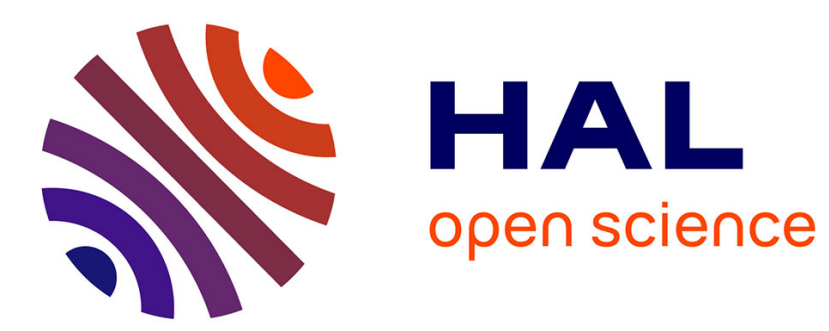

\title{
THE USE OF PHOTOEMISSION TO DETERMINE THE ELECTRONIC STRUCTURE OF SOLIDS
}

\author{
W. Spicer
}

\section{To cite this version:}

W. Spicer. THE USE OF PHOTOEMISSION TO DETERMINE THE ELECTRONIC STRUCTURE OF SOLIDS. Journal de Physique Colloques, 1973, 34 (C6), pp.C6-19-C6-33. 10.1051/jphyscol:1973607 . jpa-00215328

\section{HAL Id: jpa-00215328 https://hal.science/jpa-00215328}

Submitted on 1 Jan 1973

HAL is a multi-disciplinary open access archive for the deposit and dissemination of scientific research documents, whether they are published or not. The documents may come from teaching and research institutions in France or abroad, or from public or private research centers.
L'archive ouverte pluridisciplinaire HAL, est destinée au dépôt et à la diffusion de documents scientifiques de niveau recherche, publiés ou non, émanant des établissements d'enseignement et de recherche français ou étrangers, des laboratoires publics ou privés. 


\title{
THE USE OF PHOTOEMISSION TO DETERMINE THE ELECTRONIC STRUCTURE OF SOLIDS (*)
}

\author{
W. E. SPICER \\ Stanford University, Stanford, CA 94305, USA
}

\begin{abstract}
The use of photoemission to determine the electronic structure of solids is discussed. The three-step model of photoemission is outlined. Results from crystalline and amorphous Ge are used to illustrate a successful use of photoemission to determine electronic structure. Data from $\mathrm{Si}$ is used to illustrate the use of photoemission to investigate surface states and surface reactions. Finally, results from $\mathrm{Ni}$ and other materials are discussed to illustrate the minority of cases where difficulty remains in interpreting photoemission in terms of existing models.
\end{abstract}

1. Introduction. - Pioneering work in the use of $\mathrm{UV}$ photoemission as a spectroscopic tool was performed by Apker, Taft, Philipp and Dickey [1] in the 1950s. However, it was only in the 1960 s that this technique began to be fully developed [2], [3]. Essential to this was the simple three-step model of photoemission developed by Spicer [4], and Spicer and Berglund [3]. This model allows the optical excitation, electron transport through the solid, and escape across the surface to be treated separately. Using this approximation, a very complex phenomena is made tractable and it has proven possible for experimentalists to gain electronic structure and other information directly from their experiments. As will be emphasized later, the approximate nature of this model should always be kept in mind.

An important improvement in experimental techniques in the sixties resulted from the widespread availability of vacuum monochromators, allowing measurements to be made at photon energies much higher than the photoemission threshold. This is important in order to examine a larger range of band structure and in order to minimize difficulties due to the rapidly changing escape probability which occurs at the threshold of response.

Another key development was the arrival of vastly improved vacuum technology which made vacuum below $10^{-9}$ torr, and as low as $10^{-11}$ or $10^{-12}$ torr relatively easily available. The development of metal vacuum stations have also increased the ease with which complex operations, such as cleaving, can be performed. In signal handling an important deve-

(*) Work supported in part by the US Army Research Office-Durham and by the National Science Foundation. lopment was the application of $\mathrm{AC}$ techniques and phase sensitive detection to the photoemission experiment [5], [6]. The use of higher derivatives [7] provided a useful extension of this technique. A review has recently been written of experimental techniques for UV photoemission [7].

Normally in photoemission spectroscopy, PS, the objective is to determine the distribution in energy of the electrons excited by monochromatic radiation, $\eta(E, h v)$, and from this to deduce information about the electronic structure of the solid. Since it provides information on the optical transition probability to final states at specific energies, PS differs in a critical way from optical or X-ray techniques which, in essence, measure the total transition probability to all possible final states due to absorption of a photon of energy $h v$

$$
P(h v)=\int \eta(E, h v) \mathrm{d} E .
$$

Here $\eta(E, h)$ is more exactly defined as the probability of a transition to a final state between $E$ and $E+\mathrm{d} E$ due to absorption of a photon of energy $h \mathrm{v}$. $\eta(E, h v)$ is closely related to the external photoemission energy distribution curves (EDCs) determined experimentally.

$\eta(E, h v)$ is illustrated by figure 1 . The critical point is that it is the transition probability to a final state at a given energy $E$; whereas, $P(h v)$ is the integral over all of the possible transitions. Clearly, much detailed information is lost in going from $\eta(E, h v)$ to $P(h w)[8],[9]$.

To illustrate this more clearly, let us examine figure 2 which compares a plot of the optical constant 


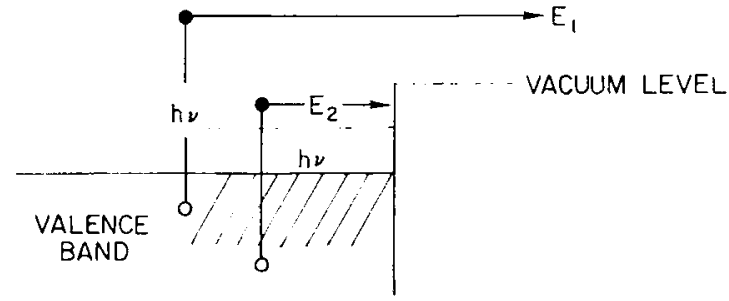

$\eta(h \nu, E) d E$ PROBABILITY $h \nu$ EXCITING ELECTRON TO FINAL STATE AT ENERGY E TO E + dE

$\epsilon_{2}--\int \eta(h \nu, E) d E$

$N(E, h \nu) d E-\cdots-\eta(h \nu, E) d E$

$N(E, h \nu)=E N E R G Y$ DISTRIBUTION OF PHOTOEMISSION

FIG. 1. - The essence of the use of photoemission to study the electronic structure of solids is determining $P(h, E)$, the probability of a photon of energy $h v$ exciting an electron to a final state at energy $E$. In contrast, it is the integral of $P(E, h v)$ over all final states which is related of the optical parameters such as $\varepsilon_{2}$.

[10] $\varepsilon_{2}$ vs. $h v$ for Ge with photoemission EDCs [11] taken at 7.8 and $8.6 \mathrm{eV}$. Note that for $h v>6 \mathrm{eV}$, there is no structure in $\varepsilon_{2}$; however, the EDCs for 7.8 and $8.6 \mathrm{eV}$ show strong structure. This structure

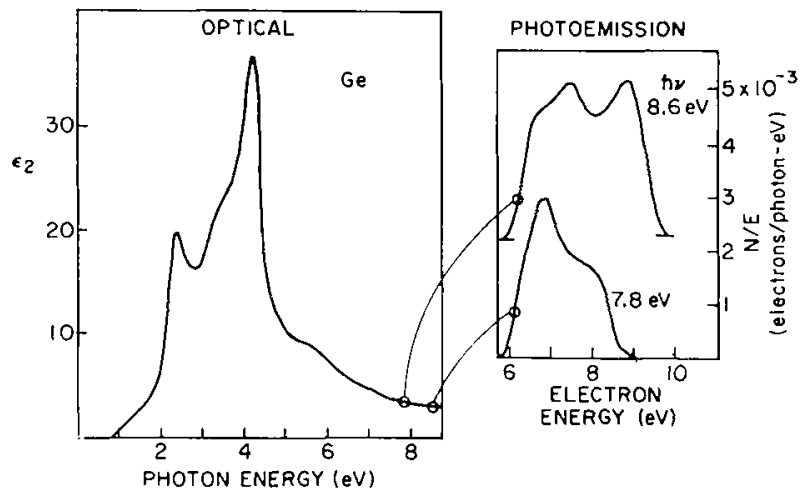

FIG. 2. - A comparison of optical and photoemission data. The left panel gives $\varepsilon_{2}$ for Ge versus $h v$. In the right hand panel, photoemission energy distribution curves (EDCs) for photon energies of 7.8 and $8.6 \mathrm{eV}$ are given. Note that there is no structure in $\varepsilon_{2}$ between 7 and $9 \mathrm{eV}$ but that the EDCs in this $h v$ range show strong structure.

changes with $h$. The EDCs give the distribution of emitted electrons as a function of their energy and thus are closely related to $\eta(E, h v)$; whereas, $\varepsilon_{2}$ is related to the integral of the transition probability per unit time over all possible final states and thus to $P(E)$. As one can see because of the integration, very little detailed information on the band structure could be gained for $h v>6.0 \mathrm{eV}$; however, the FDCs are rich in structure.

Farlier we mentioned the fact that the electrons are excited inside the solid and must move to the surface before they can escape. We have also mentioned the three step model for photoemission which has been given in detail elsewhere [3], [4], [5], [9], [12], [13]. Here, we will restrict ourselves to the essence of the transport and escape phenomena which are necessary to understand the relationship between most EDCs and $\eta(E, h v)$.

It must be realized that the separation of the photoemission event into three processes which are then treated independently is an approximation. As Schaich and Ashcraft [14] and C. Caroli, D. LedererRozenblatt, B. Roulet and D. Saint-James [15] have indicated, ideally, one would want to treat these as coupled events and, for example, take interference effects due to the surlace into account; however, this is a very difficult theoretical problem. So difficult that it has not been successfully done for any real solid. On the other hand, the three step model allows for relatively easy treatment of the three steps in which the physics is not obscure. In going to more sophisticated theoretical models, it is important that new approximations not be introduced which are less realistic in specific materials than those of the three-step model. One should expect the three-step model to becone progressively worse as the escape depth becomes shorter. Clearly, the model is in trouble if the escape depth is less than atomic spacings.

In figure 3 , we indicate schematically what happens to a component of the energy distribution between the time it is excited and the time it reaches the surface and escapes into vacuum. On the left of the figure, a hypothetical $\eta(E, h v)$ has been sketched. The details of $\eta(E, h v)$ depend on the details of the

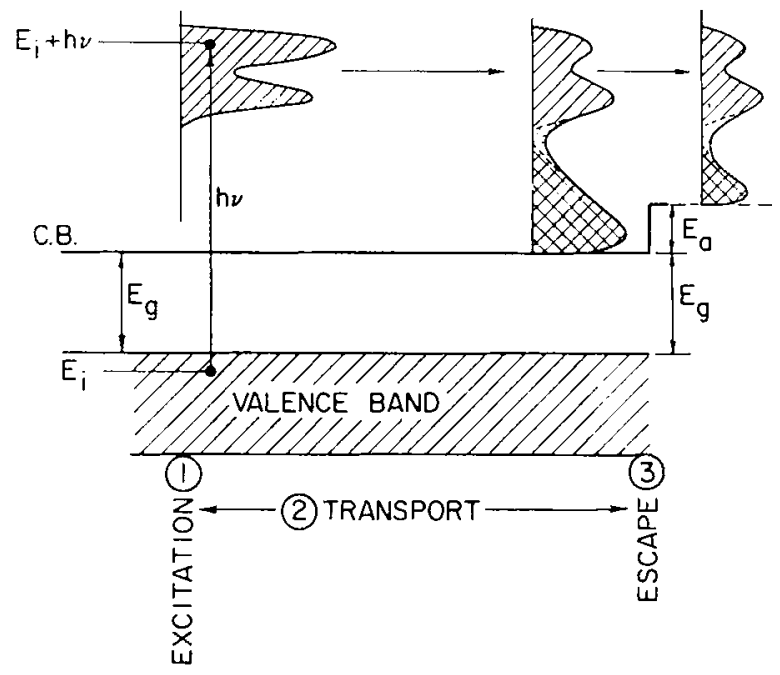

FIG. 3. - A schematic diagram illustrating the three step photoemission process. An initial optically excited distribution is shown on the left. Changes in this distribution as it approaches the surface and after it has escaped into vacuum are indicated. 
electronic structure. For example, the lower limit on $\eta(E, h v)$ is set by the width of the valence band. As they move towards the surface, electrons lose energy due to inelastic scattering [8], [9]. Electron-electron (el-el) scattering between the excited electron and the valence electrons, and electron-phonon (el-ph) scattering between the crystal lattice and the electron are the two principle events. Scattering between the electron and lattice defects or impurities is also possible.

In table I, we list the characteristics of these events. One important difference between the two scattering events is the magnitude of the energy loss. As shown in table $\mathrm{I}$, the loss in lattice scattering is small (order of $0.01-0.05 \mathrm{eV})$ compared to the excitation energy $(h v)$; whereas, in the electron-electron scattering, the loss may be a good fraction of the excitation energy; i. e., several electron volts or more.

To the first approximation, the probability, $S(E, x)$ of scattering after traveling a distance $x$ without scattering can be defined in terms of a scattering length [4], [16],

$$
S(E, x)=\exp \left(-\frac{x}{L(E)}\right) .
$$

For the phonon event, $L(E)$ is relatively independent of energy. However, as figure 4 shows, $L(E)$ is extremely strongly dependent on energy. Figure 4 also emphasizes the short (a few $\AA$ ) minimum values of $L(E)$ which occur.

Note in figure 4 that only for relatively low energies, i. e., $E \approx 6 \mathrm{eV}$, is the el-el $L(E)$ large compared with the typical el-ph scattering lengths. Only under these conditions does loss of energy to phonons become of major importance [17]. As soon as the el-el length becomes comparable to the el-ph length, el-el scattering becomes dominant due to the large energy loss per event. In this paper, we will concentrate on this case since it is most common. The reader is referred to the literature for cases where el-ph scattering dominates $[4],[16],[17]$.

In figure 3, the low energy peak which appears as the excited electrons move to the surface is due to such scattering. Because of the large energy loss associated with the el-el event, the scattered electrons are often effectively removed from the distribution or else simply provide a monotanic background. This is important because electrons are removed from the EDCs without producing new structure which would confuse the interpretation of the data. As shown in figure 3, structure due to the excitation probability, $\eta(E, h)$, remains in the EDCs after scattering and a new peak of low energy electrons is produced near the low energy cutoff of the EDC in vacuum.

To summarize, el-el scattering will remove electrons from the original distribution. This can cause broadening in structure and changes in the relative intensities

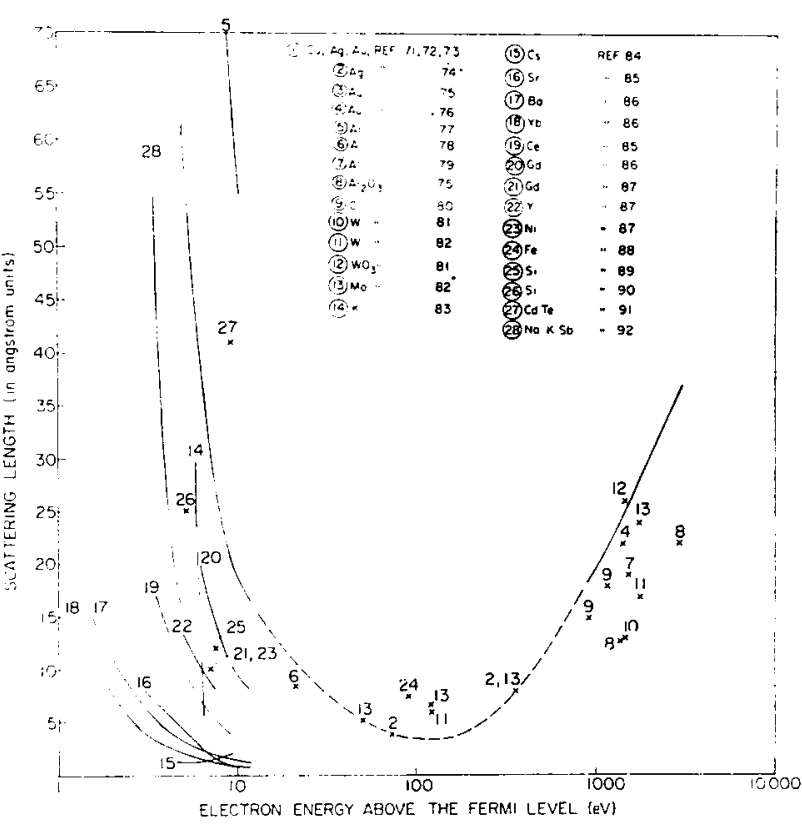

FIG. 4. - This gives a summary of the available information on electron escape depth versus photon energy. The solid curve on the right hand side of the figure is curve number 3 . The curve is taken from I. Lindau and W. E. Spicer (to be published). The references are as follows:

[71] Krolikowski, W. F. and Spicer, W. E., Phys. Rev. 185 (1969) 882 .

[72] Krolikowski, W. F. and Spicer, W. E., Phys. Rev. B 1 (1970) 478

[73] Kanter, H., Phys. Rev. B 1 (1970) 522.

[74] Palmberg, P. W. and Rhodin, T. N., J. Appl. Phys 39 (1968) 2425.

[75] Klasson, M., Hedman, J., Berndtsson, A., Nilsson, R., Nordling, C. and Melnik, P., Phys. Scripta 5 (1972) 93.

[76] Baer, Y., Hedén, P. F., Hedman, J., Klasson, M. and Nording, C., Solid State Commum. 8 (1970) 1479.

[77] Huen, T. and Wooten, F., Solid State Commun. 9 (1971) 871.

[78] Gesell, T. F. and Arakawa, E. T., Thys. Rev. Lett. 26 (1971) 377

[79] Kanter, H., Phys. Rev. B 1 (1970) 2357.

[80] Steinhardt, R. G., Hudis, J. and Perlman, M. L., Phys. Rev. B 5 (1972) 1016.

[81] Carlson, T. A. and McGuire, G. E., J. Electron Spectr. $1(1972 / 73) 161$.

[82] Tarng, M. L. and Wehner, G. K., J. Appl. Phys. 44 (1973) 1534

[83] Thomas, H., Z. Physik 147 (1957) 395.

[84] Smith, N. V. and Fisher, G. B., Phys. Rev. B 3 (1971) 3662.

[85] Helms, C. R. and Spicer, W. E. (to be published).

[86] Brodén, G., Phys. Kondens. Materie 15 (1972) 171.

[87] Eastman, D. E., Solid State Commun. 8 (1970) 41.

[88] Ridgway, J. W. T. and Hanemann, Surface Sci. 24 (1971) 451.

[89] Wagner, L. F. and Spicer, W. E. (to be published).

[90] Gobeli, G. W. and Allen, F. G., Phys. Rev. 127 (1962) 141.

[91] Shay, J. L. and Spicer, W. E., Phys. Rev. 169 (1968) 650.

[92] Splcer, W. E., J. Phys. Chem. Solids 22 (1961) 365 


\section{TABLE I}

Type of scattering

Electron-Electron

Electron-Phonon

\author{
Threshold \\ metal-none \\ non-metal-bandgap
}

none

\author{
Typical energy loss \\ (per event) \\ Large fraction of excita- \\ tion energy electron- \\ volts
}

Small (tens of meV)

\author{
Typical scattering \\ lengths \\ See figure 4 \\ can be few $\AA$
}

of peaks. Except for these changes, there is a close one-to-one relationship between structure in the measured EDCs and that in the excited distribution $\eta(E, h v)$.

Before leaving figure 4 , we should mention that the excitation function itself will in general change as one approaches the surface. Because of the termination of the lattice at the surface, new electronic states may appear and/or the electronic structure associated with the bulk will be gradually changed. This will take place in the last few atomic layers. As one can see from figure 4 , the minimum values of $L(E)$ can lie within this range of values. Thus by " tuning » $L(E)$ through selection of $E$, one has the possibility of alternately looking at bulk or surface electronic structure. Conversely, one must be careful as $h$ is varied that changes in $N(E, h v)$ due to surface effects are not attributed to changes in optical matrix elements associated with bulk excitation. The shorter the escape depth, the less meaningful it is to separate the photoemission process into excitation, transport, and escape; thus, the three step model becomes a progressively poorer approximation as the escape length becomes shorter and approaches atomic or lattice dimensions.

The last step in photoemission is the escape of the electrons across the potential barrier at the surface. The principal effect here is to cut off the low energy portion of the EDCs in such a way that the magnitude of the EDCs increase gradually from their threshold value. This is indicated in figure 3. Detailed models have been used to calculate the escape function [3], [4], [12], [13].

2. Studies of germanium. - 2.1 INTRODUCTION. A very large number of materials of all types have been studied in the last decade using photoemission. It would be quite inappropriate to attempt to review all of this work here. Rather, we will choose certain examples as illustrations of the exploration of the electronic structure of solids. The principal examples will be from germanium and silicon. These materials have been as thoroughly studied as any others. Work has been done in the X-ray as well as UV spectral range. The band structure of crystalline $\mathrm{Ge}$ and $\mathrm{Si}$ are among the best known. Interest in these materials is enhanced because they can be formed in an amorphous [19] as well as a crystalline form. Thus, the effects of long range order on the electronic structure can be studied. Surface states are of interest and these have been observed on $\mathrm{Ge}[20]$; however, much more detailed data on Si surface states [20], [21], [22] is available to the author. Therefore, Si surface state results will be discussed.

2.2 Photoemission and the band structure of CRYSTALLINE Ge. - In the recent photoemission studies of crystalline Ge [11], [23], [24], [25], there has been strong interaction between band calculations and the experimental studies. From the first, the existing band structures have formed the basis for interpreting the photoemission results, making use of $k$-conservation; conversely, as photoemission (as well as optical) experiments gave increasingly accurate locations for band structure parameters, adjustments were made in the band calculations to bring them into better agreement with experiment. We will not attempt to systematically discuss all of the Ge work, rather we will take a few representative examples.

In making connection between EDCs and band structure, one problem is that of locating structure in the EDCs in $k$ space. There is hope that direct measurements of this type can be made in the future by measuring the EDCs as a function of direction of emission of the photoelectrons ; however, this difficult technique has not yet fully proven its usefulness experimentally. Thus it is presently necessary to examine the movement of structure in EDCs with $h v$ and by comparison with the effects expected from band calculations [8], [26]. There is one set of symmetry points which can often be obtained unambiguously directly from the EDCs. This is the set located, in $k$ space, vertically above the valence band maximum (the $\Gamma$ points in Ge). Whenever $h v$ matches the energy difference between the valence band maximum and a higher $\Gamma$ point, an increased number of electrons appears near the maximum energy of the EDC [27]. In figure 5, we present the band structure and density of states of Ge. The object of the work to be presented next was to locate the second conduction band $\Gamma_{15}$. At the time there was a variation of about 1.0 to $0.5 \mathrm{eV}$ between the location of this peak in various band calculations. 

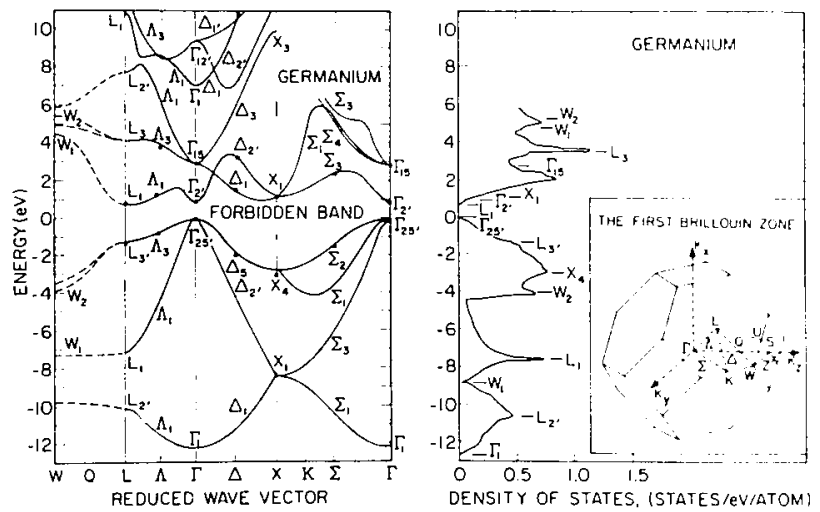

FIG. 5. - A calculated band structure and density of states for Ge.

In figure 6, EDCs are given [23] at $3.1 \mathrm{eV}$ which show a shoulder at the leading edge of the EDC due to a fully developed transition near $\Gamma_{15}$. EDCs taken at both room temperature and $80 \mathrm{~K}$ are shown. As can be seen, the shoulder becomes more pronounced at the lower temperature. From this data, one can place $3.1 \mathrm{eV}$ as an upper limit of the energy of the $\Gamma_{15}-\Gamma_{25}$ transition. To help locate the threshold with more accuracy, a series of EDCs was taken at progressively lower energies and the leading edge examined to find evidence for the first $h v$ at which the $\Gamma$ transition occurs. This data is shown in the insert to figure 6 . The change in curvature which occurs between $h v=2.9$ and $3.0 \mathrm{eV}$ places the threshold between these limits.

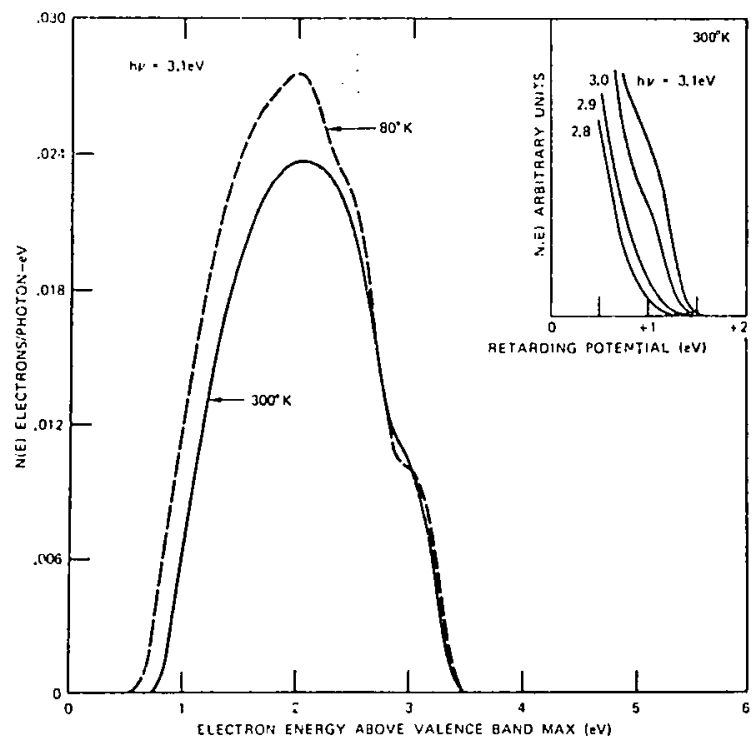

FIG. 6. - Room temperature and $80 \mathrm{~K}$ EDCs from Ge covered with a monolayer of cesium on the surface. The insert gives the leading edge of the EDCs for $2.8 \leqslant h v \leqslant 3.1 \mathrm{eV}$.

In order to locate the threshold for the transition with greater accuracy, one can electronically take the first or second derivate of the EDCs between 2.9 and $3.0 \mathrm{eV}$ and look for the point at which the curvature of the leading edge first changes. In figure 7 , we show the full EDCs at 2.9 and $3.0 \mathrm{eV}$ and first derivates of the EDC closely spaced between those values. From these curves, the threshold is placed at $2.92 \pm 0.05 \mathrm{eV}$. Careful measurements to locate the $\Gamma_{15}$ point with respect to $\Gamma_{25}$, have been made using modulated optical measurement. These located the room transitions at $2.92 \pm 0.05 \mathrm{eV}$ [23] and $2.983 \pm .004 \mathrm{eV}$, respectively. If the threshold for the latter transition is calculated taking into account line width, the threshold is found to be $2.95 \mathrm{eV}$. One sees a strong degree of agreement between the location of structure through photoemission and optical techniques.

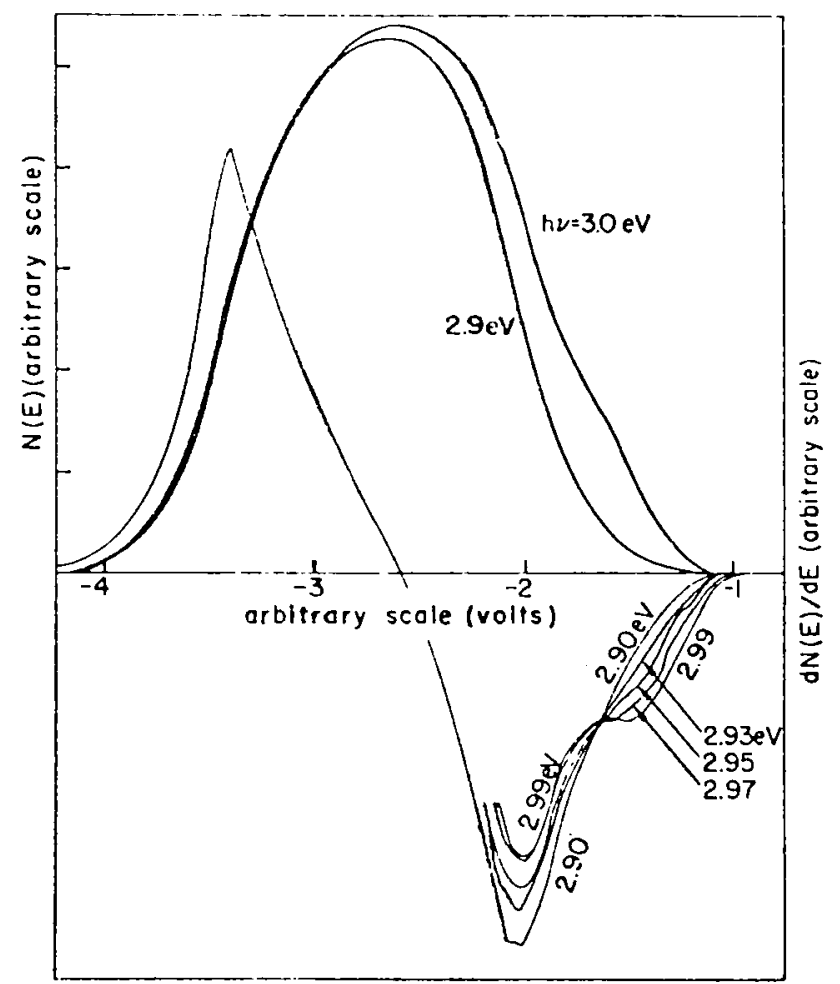

FIG. 7. - Ge EDCs at 2.9 and $3.0 \mathrm{eV}$ and the derivatives of the EDCs for $2.90 \leqslant h v \leqslant 2.99 \mathrm{eV}$.

In figure 8 , an example of the interplay between experiment and band structure is given [23], [31], [32]. Most of this plot was made before the accurate determination of the $\Gamma_{15} \Gamma_{25^{\prime}}$ separation had been made. On the abscissa is plotted the $\Gamma_{15}-\Gamma_{25}$, energy separation. On the ordinate is the energy separation between the various critical point transitions indicated by the slating lines. This plot gives the changes in these separations produced as the $\Gamma_{15}-\Gamma_{25}$, separation is varied : i. e., illustrates the effect on other symmetry point separations as $\Gamma_{15}-_{25^{\prime}}$ is varied. As can be seen, fixing $\Gamma_{15}-\Gamma_{25}$ at $3.13 \mathrm{eV}$ also has an effect on the positions of other symmetry points. The general procedure which has been followed in band calculation is one by which experimental data on symmetry point location is used to adjust the potential 


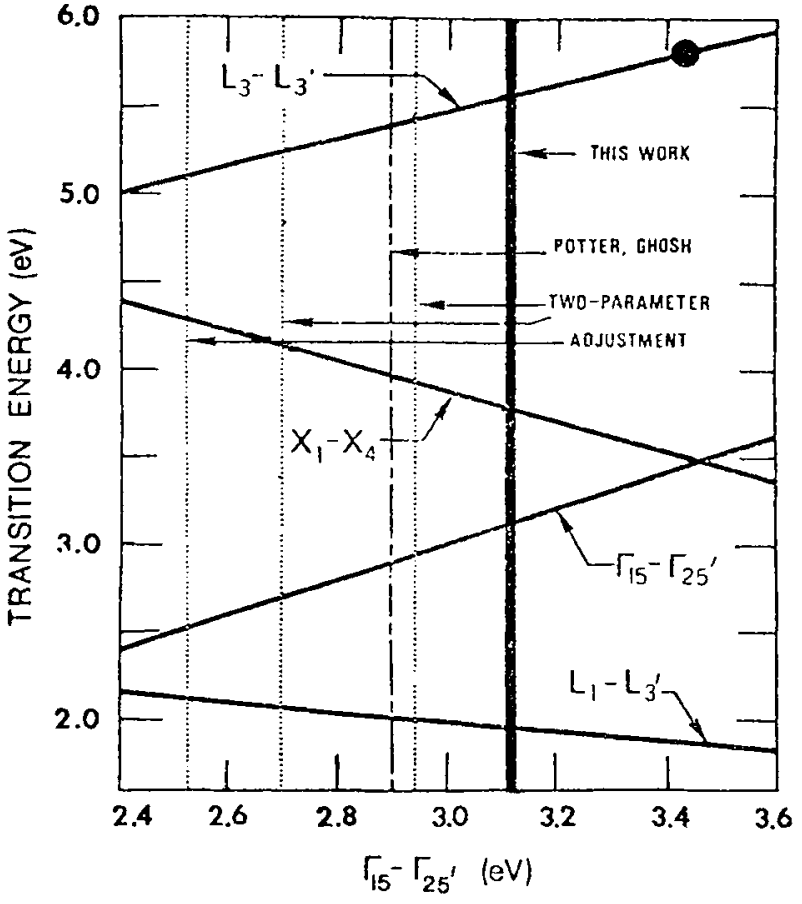

Fig. 8. - Dependence of three energy gaps on the $\Gamma_{15-}-\Gamma_{25}$, separation, from the first principles theory of Herman et al. Five choices for $\Gamma_{15}-\Gamma_{25^{\prime}}$, and their implications on other gaps, are shown as vertical lines. The three dotted lines are values predicted by three separate two-parameter adjustments. See reference [23] for further details.

used in the band structure calculations. By these means the calculations have been refined to give increasingly good fits to experimental results. Historically, the first data to which calculations were compared and adjusted was the fundamental, valence to conduction band gap. Next optical data for transitions lying within a few electron volts of the fundamental gap was used [32]. Slowly data have been obtained farther and farther from the fundamental gap and the calculations adjusted accordingly [31], [33]. As is discussed in more detail in the paper by Eastman, Freeouf and Erbudak [34], the recent extension of measurement to the lower valence bonds has necessitated further refinements in the potential [31], [33]. Later in this paper we will briefly review both the recent high energy ultraviolet and the X-ray examinations of Ge.

Next in this section, we will give examples of a series of optical transitions away from $\Gamma$ in the low $h v$ range. These are particularly important because of the conduction band information which they furnish. In figure 9, we show a series of Ge EDCs taken for energies above $3.0 \mathrm{eV}$ where the transition we saw originally at $2.93 \mathrm{eV}$ grows and moves out to the zone face at the $\mathrm{L}_{3}$. point. In the EDCs one sees the strength within about $1.5 \mathrm{eV}$ of the leading edge build up as $h w$ increases until a sharp peak is observed for $h v=5.8 \mathrm{eV}$ at a final state energy of $4.1 \mathrm{eV}$ (the zero of energy is taken at the valence band maximum). A peak also occurs in the optical response $(\omega)^{2} \varepsilon_{2}$
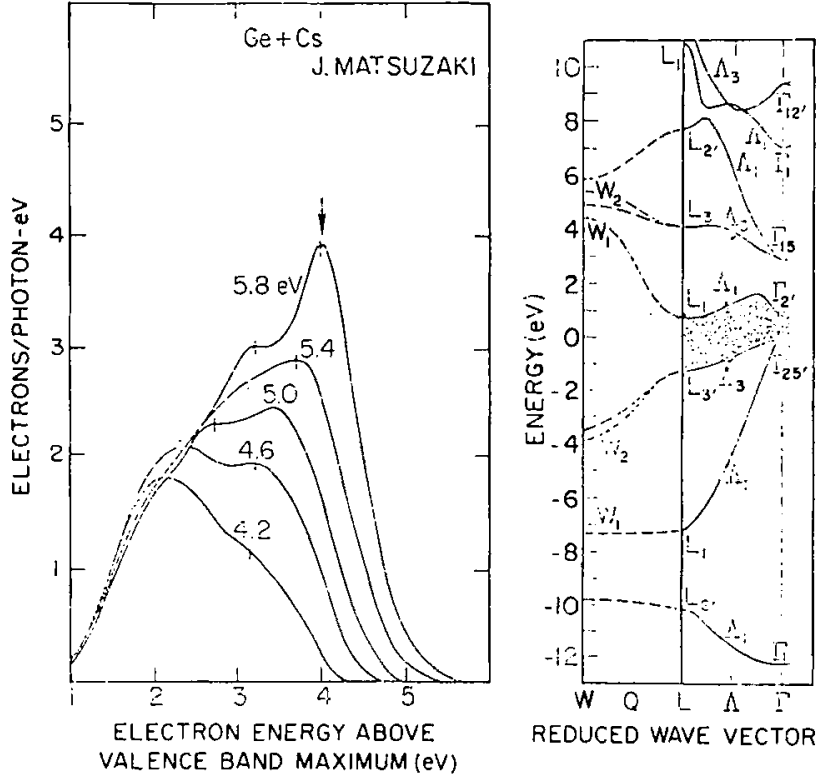

FIG. 9. - Ge EDCs for $4.2 \leqslant h v \leqslant 5.8 \mathrm{eV}$. The Ge band structure is shown on the left.

or $\varepsilon_{2}$ ) at or very near this photon energy (see Fig. 2). The increase in strength and appearance of the peak is due to the increased number of states available for direct transitions as one moves away from the center of the zone. A peak appears at the zone boundary since the bands become horizontal at this point. The peak locates the final and initial states for the $\mathrm{L}_{3}-\mathrm{L}_{3}$, transitions [11].

In figure 10 , we show the EDCs for $5.8 \leqslant h \leqslant 9.1 \mathrm{eV}$. Now the transitions move near the zone face from L to W (see the $E$ vs $\bar{k}$ diagram presented in the
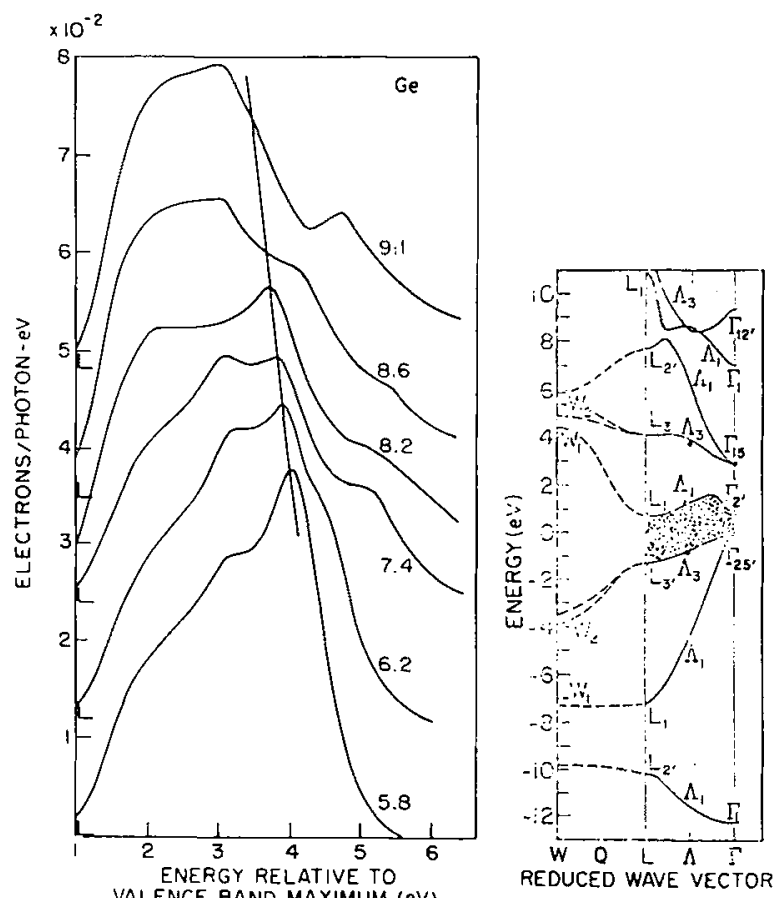

FIG. 10. - Ge EDCs for $5.8 \leqslant h v \leqslant 9.1 \mathrm{eV}$. The Ge band structure is shown on the left. 
insert). As one increases $h v$, the initial state moves down from $L$ toward $W$ and, as we see from the EDCs, the final state decreases slightly in energy. The strength of the peak decreases as $h v$ increases to $h v=7.4 \mathrm{eV}$ and then increases again to $8.2 \mathrm{eV}$. This change is due to the initial states (see the figure) and is due to the fact that the number of states available for the transition should be inversely proportional to the slope of the $E$ vs $k$ curve. As can be seen from the band diagram, the intensity should be a maximum near the $W$ and $L$ points and a minimum approximately midway between them.

The most striking feature of the data is the abrupt disappearance of the peak between $h v=8.2$ and $8.6 \mathrm{eV}$. This peak disappears because the bottom of the first set of valence bands has been reached. Thus from the data of figure 10, we locate the bottom of the band $\left(\mathrm{W}_{2}, \Sigma_{1} \mathrm{~min}\right)$ at $-4.5 \pm 0.3 \mathrm{eV}$ and produce considerable detailed information about the bands near the zone face.

It is not apparent from the band diagram why the energy of the peak should decrease slightly as $h v$ goes from 5.8 to $8.2 \mathrm{eV}$. Because of this, it is clear that more work should be done on the final band structure involved in these transitions. However, if one examines the density of states of figure 5, one sees that the peak in the density of states near $\mathrm{L}_{3}$ occurs slightly below the $\mathrm{L}_{3}$ point. Thus the majority of states must occur below $\mathrm{L}_{3}$ and off the symmetry axes shown in the $E$ vs $k$ diagrams.

It is also apparent that a peak in the final density of states is being seen in the photoemission data. Only if this were the case would the peak near a final state energy of $4 \mathrm{eV}$ remain over such a wide photon energy range. Similar structure has been seen in a number of other semiconductors [8], [27], [28], [29]. However, no such clear cut evidence occurs in metals where the final state structure is due to d-bands.

We have discussed here only a few of the critical points whose position have been determined through PS. A more complete description of the Ge will be published elsewhere [11]. Further details of the analysis of UV data has been given previously [8].

In table II, we give a summary of critical points located in this way for Ge [11] and compare them to the results of various band calculations. Several comments can be made about the data in table II. First, the agreement with the 1973 calculations [25], [31], [33] is markedly better than for the 1966 pseudopotential calculation [35]. This is clearly due to the use of more sophisticated potentials in the recent work and to the fitting to more complete experimental data which extends over a wider energy range; however, it should be noted that the adjusted first principles calculation of Herman et al. [36] of 1967 is markedly better in some regards than the 1966 pseudo-potential calculation, e. g. at $\mathrm{L}_{3}, \mathrm{~W}_{2}, \Sigma_{1}$ (min) and the conduction band points lying above $L_{3}$.

Recently, Ge has been studied using both X-ray photoemission and higher energy ultraviolet synchrotron radiation. Here we would like to present Ge photoemission data taken at the high photon energies in order to compare the results of these studies with those from the studies using $h v<12 \mathrm{eV}$ just described.

In figure 11, we reproduced curves from the work of Grobman and Eastman [20]. In the upper panel, one has the results of band calculations. One curve gives the calculated valence band density of states as well as EDC calculated assuming direct transitions for $h v=25 \mathrm{eV}$. The effect of scattering has been included in these curves. In the lower panel one has the measured EDC. As can be seen, the measurement

TABLE II

Ge results from low energy UPS spectra

$\begin{array}{cccccc}\begin{array}{c}\text { Energy } \\ \text { level }\end{array} & \begin{array}{c}\text { Transition } \\ \text { observed }\end{array} & \text { UPS (DMS) } & \text { CB } & \begin{array}{c}\text { Ph } \\ \text { PP }\end{array} & \text { CHO } \\ - & - & -1966 & 1973 & 1973 \\ \Gamma_{15} & \Gamma_{15}-\Gamma_{25^{\prime}} & 3.14 \pm 0.05 & - & - & - \\ \mathrm{L}_{3} & \mathrm{~L}_{3}-\mathrm{L}_{3^{\prime}} & 4.1 \pm 0.3 & 4.3 & 4.3 & 4.1 \\ \mathrm{~L}_{3^{\prime}} & \mathrm{L}_{3}-\mathrm{L}_{3^{\prime}} & -1.7 \pm 0.3 & -1.1 & -1.4 & -1.3 \\ \mathrm{~W}_{2}, \Sigma_{1}(\mathrm{~m} \mathrm{In}) & & -4.5 \pm 0.3 & -3.8 & -4.55 & -4.5 \\ \mathrm{X}_{4} & \mathrm{X}_{3}-\mathrm{X}_{4} & -3.1 \pm 0.3 & -2.4 & -3.2 & -3.0 \\ \mathrm{X}_{3} & \mathrm{X}_{3}-\mathrm{X}_{4} & 8.3 \pm 0.3 & - & - & 10(1967)\end{array}$

Zero of energy taken at valence band maximum $\left(\Gamma_{25^{\circ}}\right)$.

DMS = Donovan T., MATSUzaki J. and Spicer W. B., to be published.

CB = Cohen M. L. and Bergstresser T. K., Phys. Rev. 141 (1966) 789.

PP = Phillips J. C. and Pande K. C., Phys. Rev. Lett. 30 (1973) 787.

CHO = Chekroun C. D., Ortenberger I. B. and Herman F., to be published or (for $\mathrm{X}_{3}$ and $\Gamma_{1}$ ) Herman F., Kortun R. L., Kuglin C. D. and Shay J. L., Proc. II-VI Conf., ed. D. G. Thomas (W. A. Benjamin, N Y, 1967). 


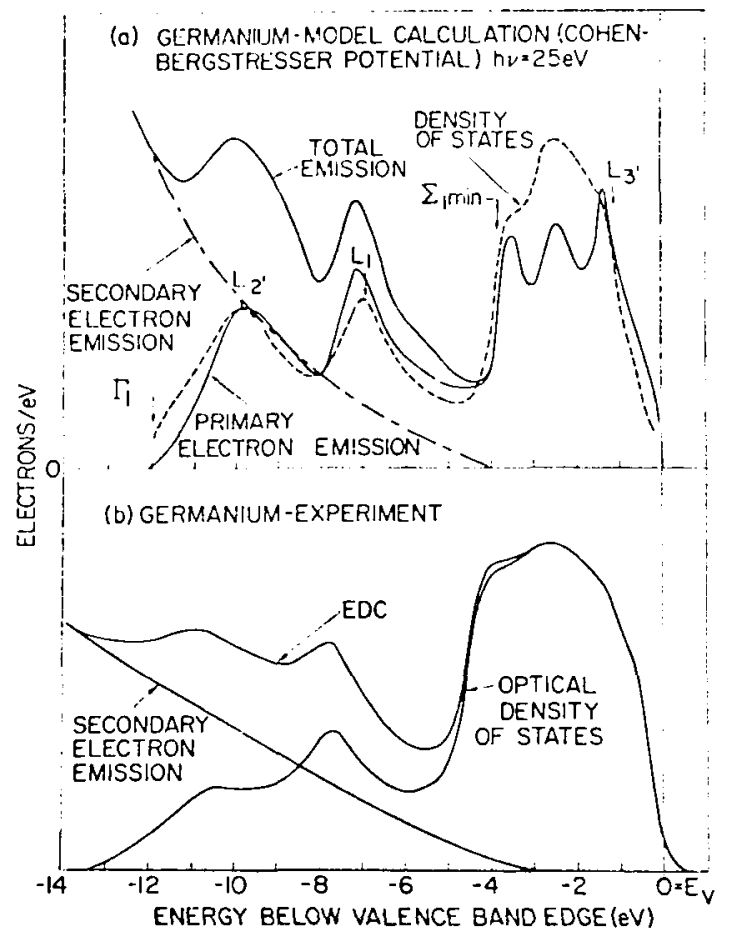

Fig. 11. - Results from Grobman and Eastman (Ref. [24]) for $h v=25 \mathrm{eV}$. The upper panel shows an EDC calculated on the basis of direct transitions (solid curve) and the calculated density of states (dashed curve). The lower panel shows the measured EDC.

resembles the valence band density of states much more closely than the direct transition calculation. Based on this, Grobman and Eastman have suggested that for approximately $h v \geqslant 25 \mathrm{eV}$ the energy distributions of $\mathrm{Ge}$ reflect the valence density of states more closely than an energy distribution due to direct transitions. This result is a little surprising and it may be due to the fact that the escape length becomes very short (perhaps $5-10 \AA$ ) at $25 \mathrm{eV}$.

The valence band of $\mathrm{Ge}$ has also been examined by Shirley [37] using X-ray photoemission with $1486.6 \mathrm{eV}$ photons. A curve for crystalline $\mathrm{Ge}$ is presented in the upper panel of figure 12. A curve for amorphous $\mathrm{Ge}$ is presented in the lower panel. This will be discussed later. As can be seen from both figures 11 and 12, using higher energy one examines the complete valence band, not just the upper $5 \mathrm{eV}$ or so examined with $h v<12 \mathrm{eV}$. However, these experiments do not give the information on the conduction bands obtained with $h v<12 \mathrm{eV}$. In fact they appear to give no conduction band information.

In table III, the critical points found by the various experiments as well as calculated values are shown. It is noted that the XPS values for $\mathrm{W}_{2}, \Sigma_{1}$ (min) and $\mathrm{X}_{4}$ differ from the UPS results and recent band calculations by about an electron volt. It is suggested that this difference is due to the difficulty of precisely determining critical points in XPS where because of the low resolution (0.5-1.5 eV), structure in the density of states is difficult to locate. This makes for special difficulties in the first set of valence bands $(E>-4.5 \mathrm{eV})$ where there are a number of critical points spaced fairly closely, none of which completely dominates the density of states. The difference between

TABLE III

Comparison of UPS, XPS and calculated critical point energies in Ge

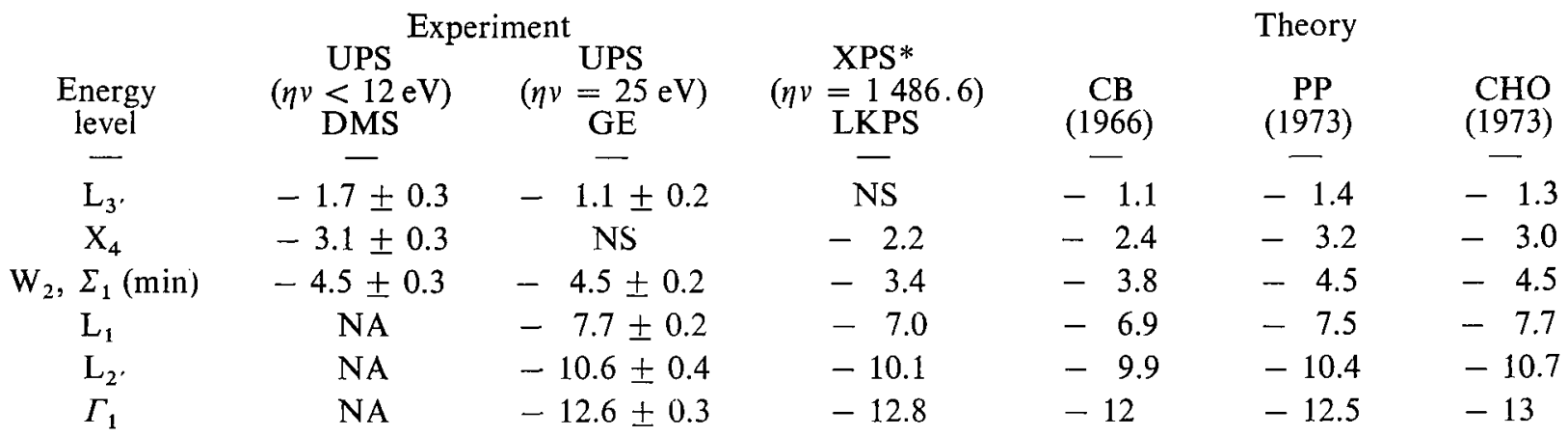

Zero of energy at valence band maximum $\left(\Gamma_{25^{\circ}}\right)$.

* Binding Energy has been decreased by $0.2 \mathrm{eV}$, to refer it to the valence band maximum.

NS $=$ Not Seen $\quad \mathrm{NA}=$ Not Accessible.

DMS = Donovan T., MATSUZAKi J. and SPICER W. E., to be published.

GE = Grobman W. D. and Eastman D. E., Phys. Rev. Lett. 29 (1972) 1508.

LKPS = Ley L., KowalczyK S., Pollack R. and Shirley D. A., Phys. Rev. Lett. 29 (1972) 1088.

$\mathrm{CB}=$ Cohen M. L. and Bergstresser T. K., Phys. Rev. 141 (1966) 789.

$\mathrm{PP}=$ Phillips J. C. and Pande K. C., Phys. Rev. Lett. 30 (1973) 787.

CHO = Chekroun C. D., Ortenberger I. B. and Herman F., to be published or (for $\mathrm{X}_{3}$ and $\Gamma_{1}$ ), Herman F., Kortun R. L., Kuglin C. D. and Shay J. L., Proc. II-VI Conf., ed. D. G. Thomas (W. A. Benjamin, N Y, 1967). 


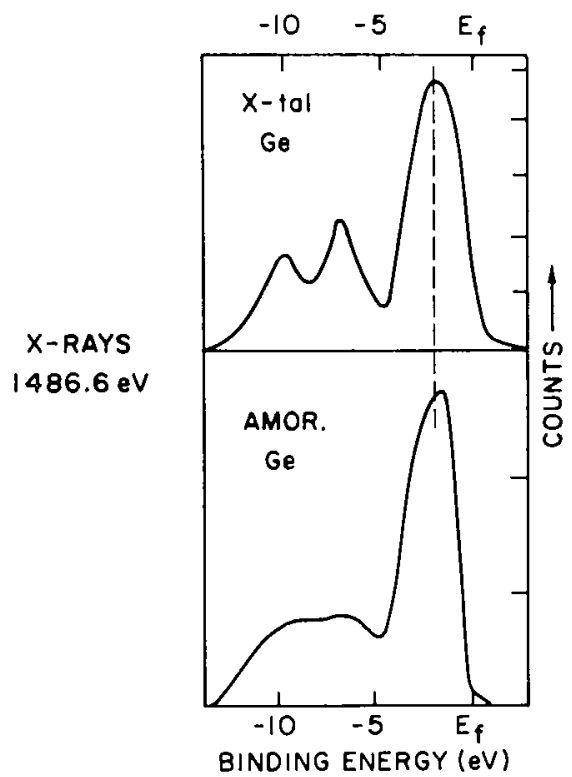

FIG. 12. - X-ray photoemission results from crystalline and amorphous Ge.

the UPS and XPS results is not so large (about half an $\mathrm{eV})$ for the low valence bands $\left(\mathrm{L}_{1}, \mathrm{~L}_{2}\right.$, and $\Gamma_{1}$ critical points), since here the bands are simple and sharper peaks appear corresponding to two of the critical points.

There is an interesting disagreement of $0.6 \mathrm{eV}$ between the UPS results for the location $L_{3}$. This may be partially due to the fact that $\mathrm{L}_{3}$, does not produce strong structure in the density of states so that it is hard to locate for $h v=25 \mathrm{eV}$. The same effects that remove the $k$ conserving condition at $h v=25 \mathrm{eV}$ may broaden the EDCs making it more difficult to locate $\mathrm{L}_{3}$ '.

To summarize, the band structure of $\mathrm{Ge}$ has been studied in three photon energy ranges using photoemission. The results obtained in the three different sets of experiments agree reasonably well and are consistant with results from band calculations. The differences found seem to have reasonable explanations. Thus it appears that the three step model works reasonably well for $\mathrm{Ge}$ from the visible into the $\mathrm{X}$-ray spectral regions.

2.3 Amorphous Ge. - One of the fascinating things about $\mathrm{Ge}$ is that it exists in both the crystalline and amorphous phases [19]. In the amorphous phase, it retains the covalent bond ; i. e., the chemical bonding scheme remains unchanged but looses long-range order. Thus, the study of amorphous and crystalline $\mathrm{Ge}$ is particularly interesting since it provides a method for separating out to some extent the effects of long-range crystalline order and the effects of local, chemical bonding due to the covalent bond [38].

For the general purpose of illustrating the use of photoemission to study the electronic structure of solids, the study of amorphous $\mathrm{Ge}$ is instructive since it shows the way photoemission can be used when there is no prior knowledge of the electronic structure. This is in contrast to the studies of the crystalline material where prior knowledge of the band structure was important in interpreting the photoemission data.

In figure 13, we compare EDCs from a Ge single crystal and an amorphous film prepared by evaporation [39]. As can be seen, the two sets of data are markedly different. The crystalline data is characterized by a number of pieces of rather sharp structure which move with energy in peculiar ways. This is due to the $k$-conservation condition and the details of the band structure.
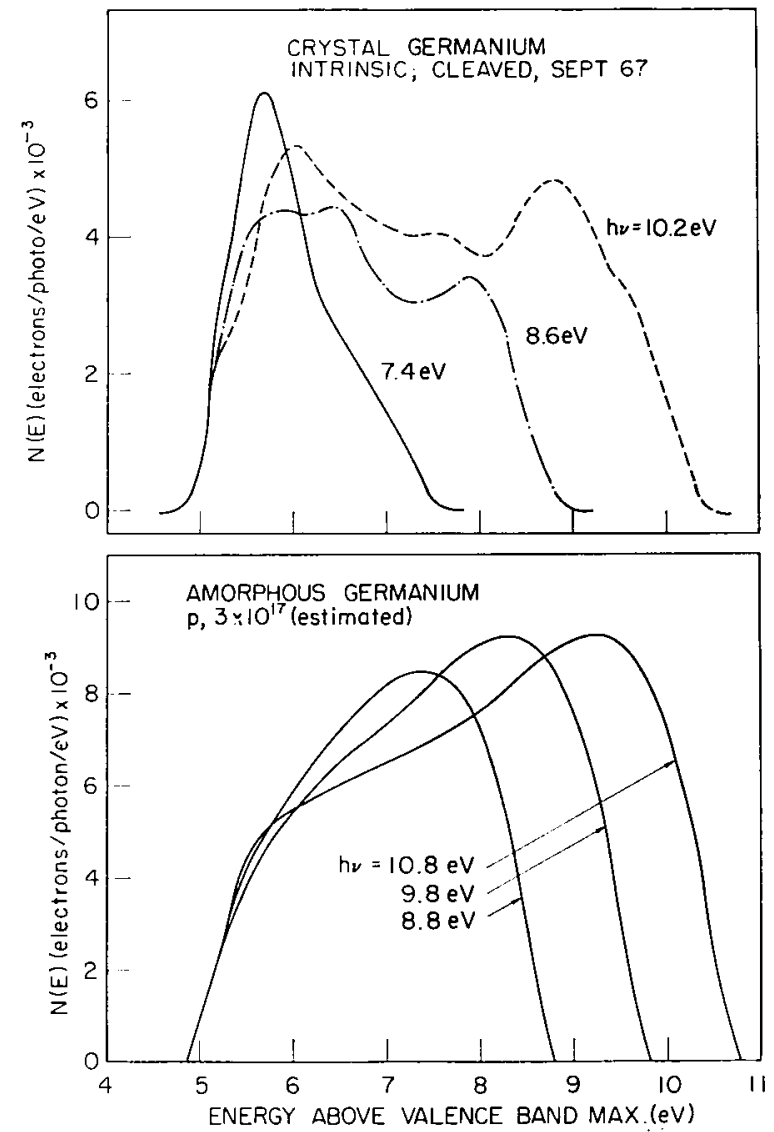

FIG. 13. - A comparison of ultraviolet EDCs from crystalline and amorphous $\mathrm{Ge}$.

In contrast, the data from the amorphous material shows very little structure ; just a single broad peak which moves monatonically to higher energy with increasing photon energy. This behavior suggests that the structure originates from the valence band. To test this hypothesis, the EDCs are plotted in figure 14 against the energy of the state from which they are excited. This is done by simply subtracting the photon energy from the final state energy $\left(E_{\mathrm{i}}=E-h v\right)$. The superposition of the structure on an initial state plot gives strong evidence that it is due to structure in the initial density of states. 


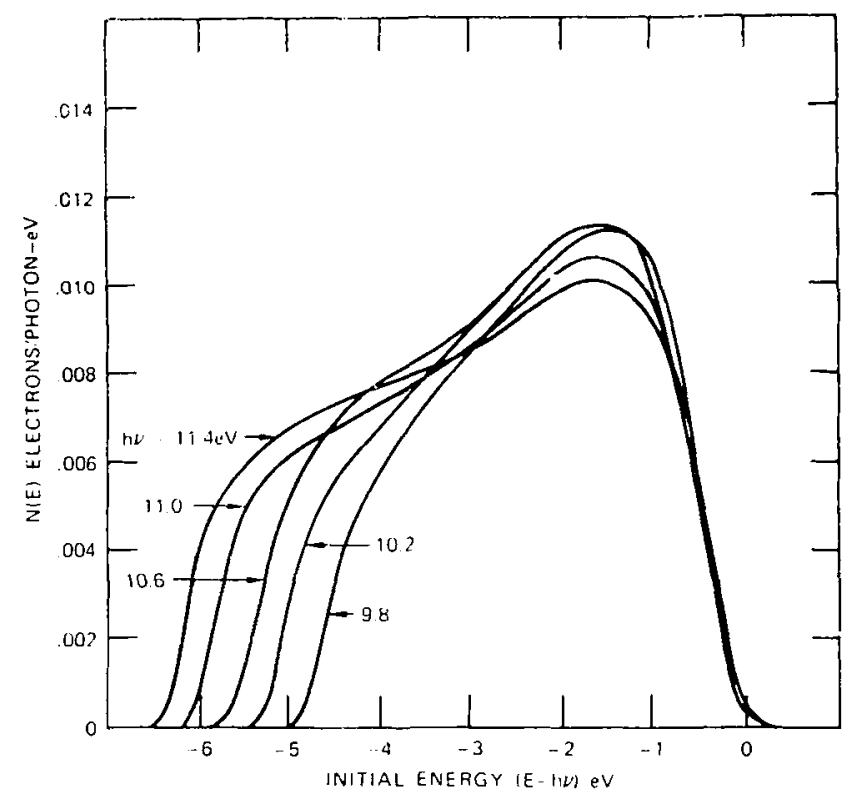

FIG. 14. - EDCs from crystalline Ge plotted versus the initial energy of the state from which the electrons were excited.

The behavior of the amorphous EDCs suggests optical transitions which depend primarily on the density of states and conservation of energy. The non-direct model is outlined in table IV. In using the non-direct model to analyze photoemission data, the object is to deconvolute the EDCs to obtain $N_{\mathrm{C}}(E)$ and $N_{\mathrm{v}}(E)$, the conduction and valence band optical density of states. Details for this procedure have been given elsewhere [3], [12], [39]. Suffice to say here that it is a self consistant procedure which takes into account electron loss due to electron-electron scattering. Thus, the EDCs are related to $P(E, h v)$ and the density of states products.

\section{TABLE IV}

\section{Non-direct model}

\section{(1) $\bar{k}$ Conservation Not Important}

$\Im(E, h v) \propto M^{2}(E, E-h v) N_{\mathrm{c}}(E) N_{\mathrm{v}}(E-h v)$.

(2) Constant Matrix Element Approximation (Limited $h v$ Range).

$$
\begin{gathered}
\mathfrak{T}(E, h v) \propto N_{\mathrm{c}}(E) N_{\mathrm{v}}(E-h v) \\
\varepsilon_{2} \propto \int \uparrow(E, h v) \mathrm{d} E=\int N_{\mathrm{c}}(E) N_{\mathrm{v}}(E-h v) \mathrm{d} E .
\end{gathered}
$$

Figure 15 shows the optical density of states obtained for amorphous $\mathrm{Ge}$ using both the photoemission and optical data. It is necessary to use optical data to obtain the lowest one or two $\mathrm{eV}$ of the conduction band, since this lies under the vacuum level and cannot be examined by photoemission [39].

Once the optical density of states has been determined, the method of analysis can be used in reverse

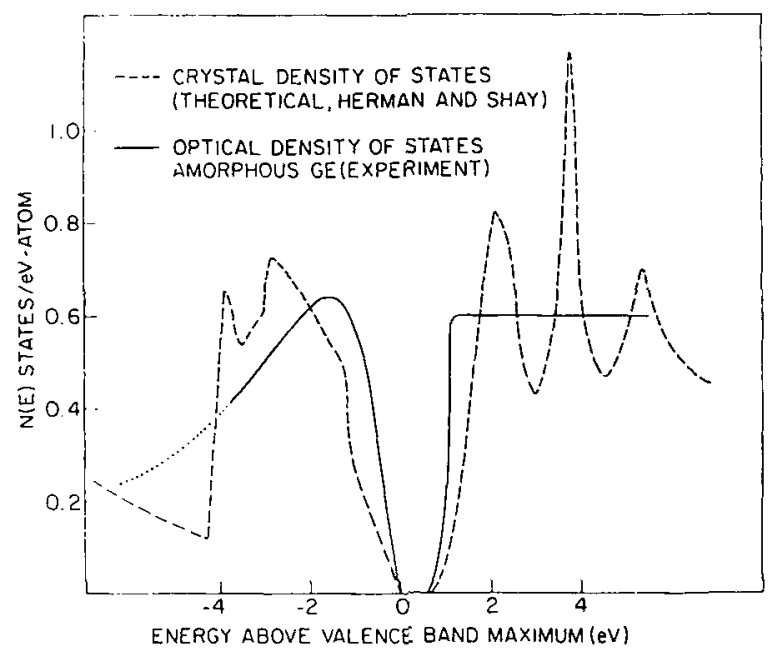

Fig. 15. - Density of states for amorphous and crystalline Ge. The amorphous optical density of states was obtained from optical and photoemission experiments. The crystalline density of states is due to band calculations.

order and the EDC as well as the optical constant $\varepsilon_{2}$ can be calculated and compared to experiment. Since there are a number (15 to 40 ) of EDCs, the system is strongly over determined and these calculations give a strong test of the analysis. In figure 16, we present typical EDCs compared to experiment [39]. In figure 15 , the crystalline and amorphous densities of states are shown [38], [39]. Note that the sharp structure in the crystalline densities of states is gone in the amorphous material. Direct evidence is found in the photoemission data for much of the sharp structure in the crystalline densities of states, for example the peak at $\mathbf{L}_{3}$. (See the previous section.)

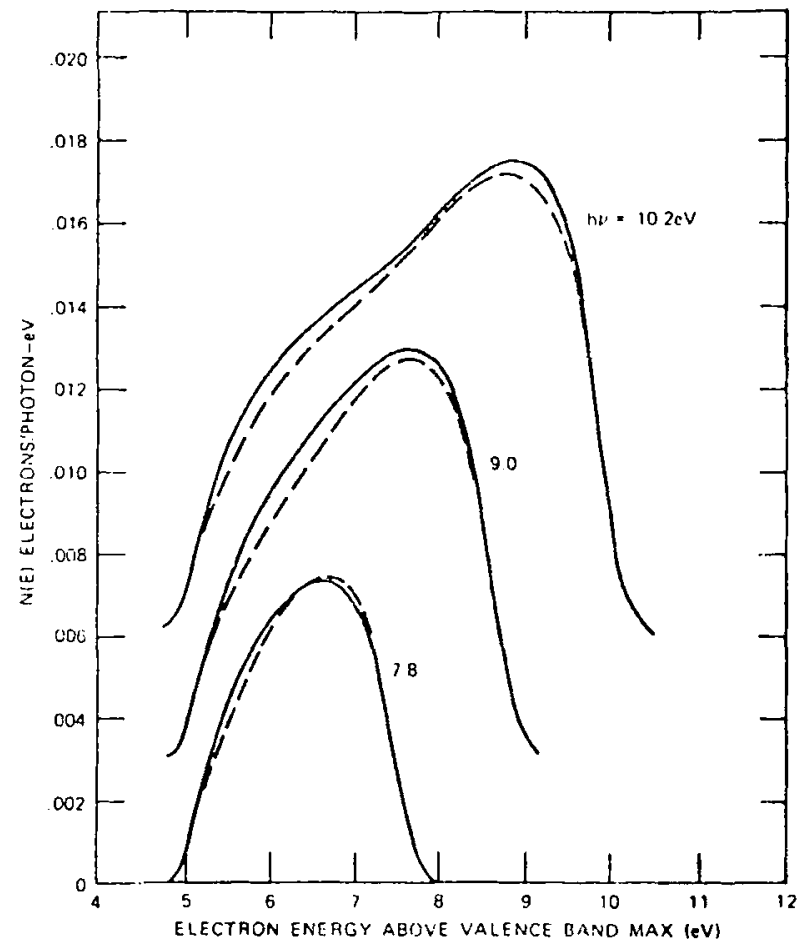

Fig. 16. - Calculated (dashed curve) and measured (solid curve) EDCs from amorphous Ge. 
The amorphous Ge study is an example of how photoemission and optical data can be combined to provide an estimate of the electronic structure of a solid in a case where no theoretical calculations are available. Features of the density of states shown in figure 15 have been confirmed by recent XPS [37] (the valence band) and soft $\mathrm{X}$-ray absorption experiments [40]. A detailed discussion of the reliability of the amorphous density of states has been given elsewhere.

In the lower portion of figure 12 , we present the $\mathrm{X}$-ray results obtained from amorphous $\mathrm{Ge}$ by Shirley [37]. The portion above $-5 \mathrm{eV}$ is consistant with figure 15 . The lower parts of the valence bands were not seen by the low energy UV experiments and so are of particular interest. Note that the crystalline material has a double peak structure between - 11 and $-6 \mathrm{eV}$. The two peaks lose their identity in the amorphous material. Wearie and Thorpe [41] have explained this in terms of the occurrence of odd numbered rings and six membered rings in the amorphous material (the crystalline material contains only six membered rings).

It is interesting to speculate as to the reason for the loss of structure above $-5 \mathrm{eV}$ in the conduction and valence bands of amorphous Ge. It is likely that this is due to the loss of the Bragg reflection condition in the amorphous material [38]. The structure in the crystalline material can be thought of as being due to the combined effect of the chemical (covalent) band and the Bragg reflection condition (the latter condition giving rise to the Brillouin zones and Van Hove singularities). In the amorphous material, the Bragg reflections are gone and the effects of the chemical bonding alone produce the observed density of states.

3. Surface states on crystalline Ge and Si. - As one approaches the surface of a solid, the electron structure must be affected by the termination of the solid. For the covalent semiconductors $\mathrm{Si}$ and $\mathrm{Ge}$, it has been rather well established that this termination produces a new set of states associated with the surface. (The "normal " band structure may also be modified just at the surface.) These have recently been detected over their full extent using UPS. Wagner and Spicer [21] and Eastmand and Grobman [20] have reported surface states on $\mathrm{Si}$ and Eastman and Grobman [20] on Ge and GaAs. Here we will discuss the results for $\mathrm{Si}$ which have been obtained from rather detailed studies by Wagner and Spicer [21].

The principal problem is that of distinguishing the surface states from bulk states. Three tests for this have been used in the work of Wagner and Spicer :

1) The photon energy dependence of position of the structure in EDCs.

2) Sensitivity to surface contamination.

3) Effect of band bending.
In figure 17, EDCs are given from $10^{20} / \mathrm{cm}^{3}$ n-type Si cleaved in a vacuum of less than $10^{-11}$ torr. Two types of structure are seen :

1) that below - $1.5 \mathrm{eV}$ which moves in initial energy as $h v$ is varied, and

2) that above - $1.5 \mathrm{eV}$ maintains an initial state energy independent of $h v$.

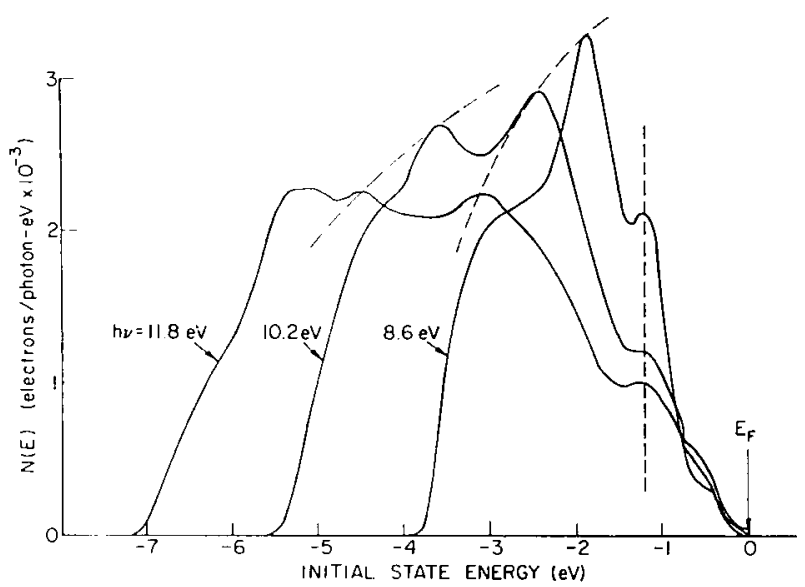

FIG. 17. - EDCs from single crystal Si cleaved in ultra high vacuum $\left(p<10^{-11}\right.$ torr). The peak near $-1.2 \mathrm{eV}$ is due to surface states.

The movement of the first group of structure is, due to $k$-conservation and the details of the band structure. The observed structure can be understood [22] (just as the case of $\mathrm{Ge}$ discussed in the last section) in terms of that band structure. The structure above $-2.5 \mathrm{eV}$ cannot be understood in terms

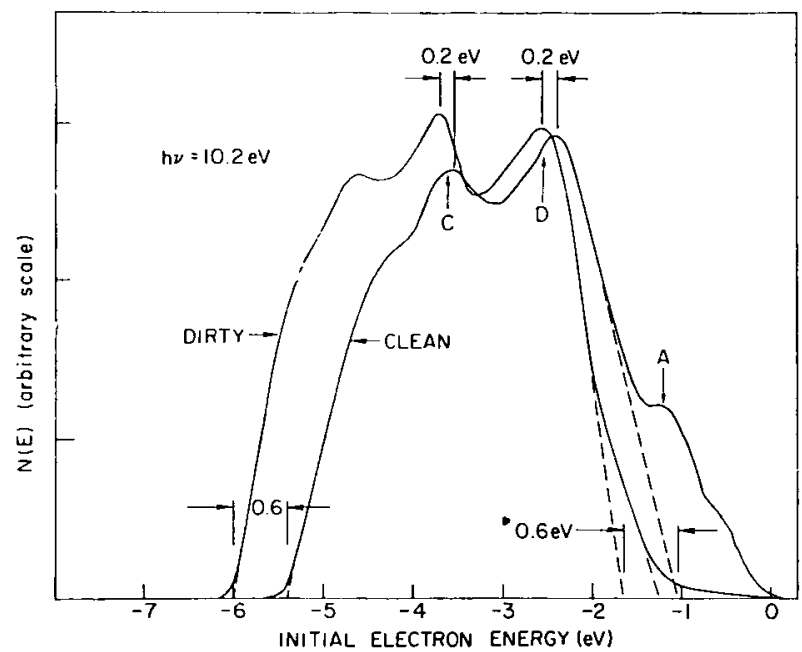

Fig. 18. - EDCs from Si before and after contamination by approximately a monolayer of gas at a pressure of about $10^{-10}$ torr. Note the disappearance of the surface states (peak A). The shift in the low-energy cut off of the curve is due to a change in work function. The peaks $\mathrm{C}$ and $\mathrm{D}$ shift by approximately $0.2 \mathrm{eV}$ due to the change in Fermi level pinning by the surface states. 
of the bulk band structure. Its behavior is consistant with excitation from a fairly narrow $(1.5-2.0 \mathrm{eV}$ wide) band of surface states. More detailed discussion is given elsewhere [20], [21], [42].

The states above $-2.5 \mathrm{eV}$ were stable for many weeks in pressures less than $10^{-11}$ torr. However, they disappeared in a period of hours at pressures of about $5 \times 10^{-10}$ torr. In figure 18 , we indicate the changes produced in the EDC by such exposure. The surface states at the leading edge of the curve have completely disappeared whereas the two bulk peaks are essentially unchanged in intensity. These peaks are shifted slightly because of a change in the pinning of the Fermi level at the surface. The lower edge of the EDCs is completely shifted exposing additional structure. This shift is due to a change in work function produced by the surface contamination. Next, consider the effects of band bending. In figure 19, we indicate the changes expected in band bending on going from $10^{20} / \mathrm{cm}^{3}$ n-to $10^{20} / \mathrm{cm}^{3}$ p-type Si. Note that the position of bulk states may be shifted by as much as the band gap energy relative to the Fermi level as the doping is changed from degenerate $p$ to degenerate $n$. The band bending takes place over approximately $30 \AA$ for a doping of $10^{20} / \mathrm{cm}^{3}$; whereas, the escape length appears to be as short as $12 \AA$ for excited electrons with energies of approximately $11 \mathrm{eV}$ in this material. (See Fig. 4 for escape depth data on other materials.) If the escape depth is shorter than the band bending distance, the shift in energy of the bulk state seen in the EDCs will be less than the complete band gap. However, the shift in the surface states will be much less. It will result from the movement of the Fermi level through the surface states.

Figure 19 gives EDCs taken for $10^{20} / \mathrm{cm}^{3} \mathrm{n}$ - and p-type samples and an almost intrinsic n-type sample.

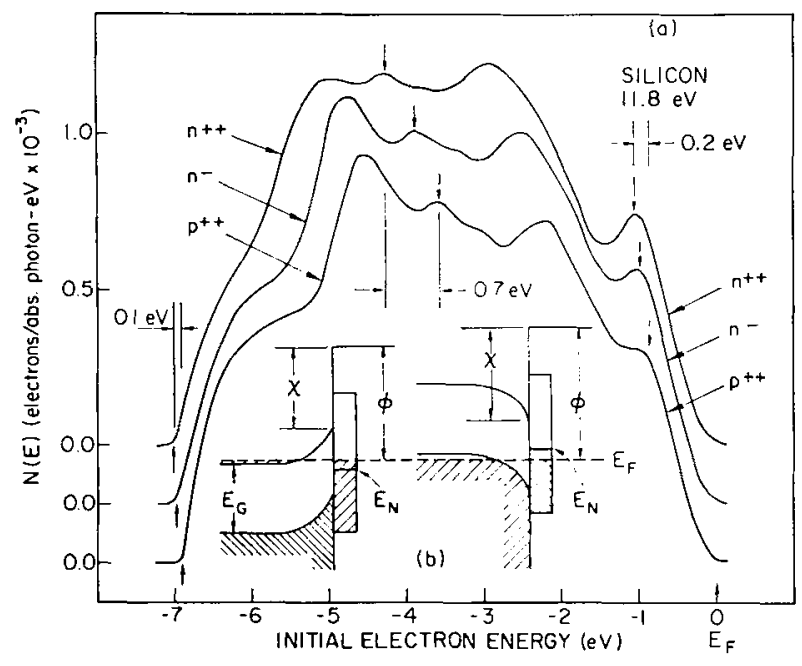

Fig. 19. - EDCs for $10^{20}$ doped $n$ and $p$ type silicon as well as for an almost intrinsic $n$ sample $\left(\mathrm{n}^{-}\right)$. The insert indicates the change in band bending due to bulk doping. The examples are for heavily coped $n$ and $p$ type material.
As can be seen, the bulk structure shifts by $0.7 \mathrm{eV}$; whereas the surface states shift by only about $0.2 \mathrm{eV}$.

As seen from the above, all three tests indicate that the states observed near the leading edge of the EDCs are surface states. In figure 20, the density of filled states deduced in this work is indicated. The total density corresponds to roughly one surface state per surface atom.

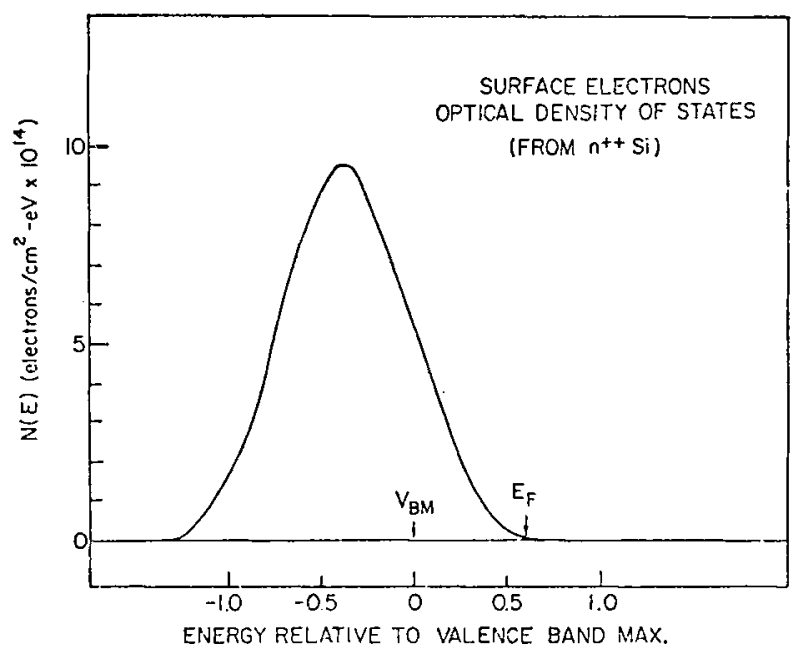

FIG. 20, - A sketch of the density of surface states on clean Si.

Further and better controlled studies have been made on the effect of a foreign gas on the surface states. In these studies, the surface of a freshly cleaved Si crystal was exposed to controlled amounts of pure $\mathrm{O}_{2}$ gas. In figure 21 we show EDCs obtained after various exposures. The exposures are given in terms of Langmuirs, L. A Langmuir is approximately the exposure to produce a monolayer coverage if all arriving gas were to stick to the surface. As can be seen, the surface states preferentially disappear as the oxygen exposure increases. They are reduced

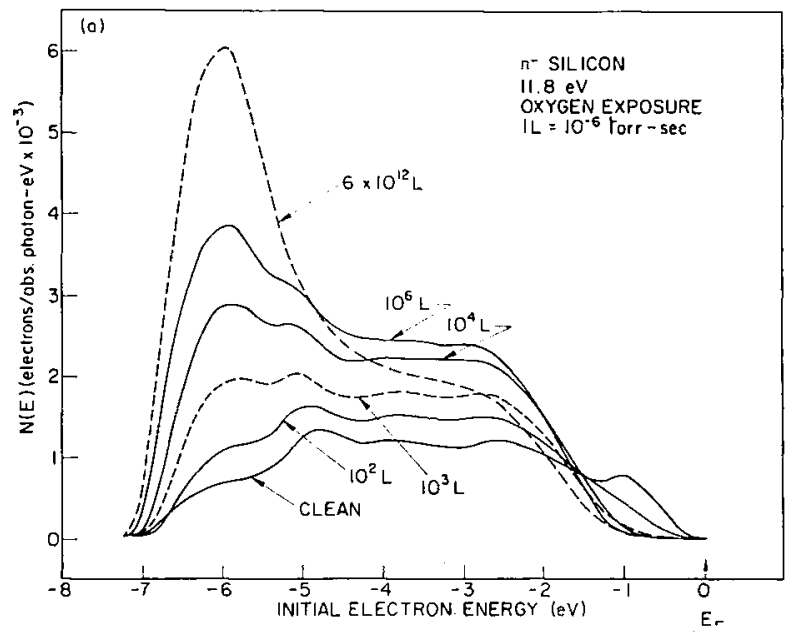

FIG. 21. - The effect of oxygen exposure on Si EDCs. Exposure is given in Langmuirs (L). Note the disappearance of the surface states and the appearance of a new Silicon oxide peak as oxygen exposure is increased. 
by about a factor of 100 after $10^{3} \mathrm{~L}$ exposure. The sticking probability on this type of surface is thought to be about $10^{-3}$, therefore this represents roughly a monolayer coverage. Note that the overall height of the bulk photoemission increases with oxygen coverage. This indicates that the escape probability is increasing.

As the oxygen exposure increases, a new peak builds up on the low energy side of the EDC. This is due to photoemission from silicon oxide. In figure 22 we show EDCs from silicon exposed to $6 \times 10^{12} \mathrm{~L}$ of oxygen and from thermally grown $\mathrm{SiO}_{2}$ [43]. As can be seen, there is agreement between the two curves.

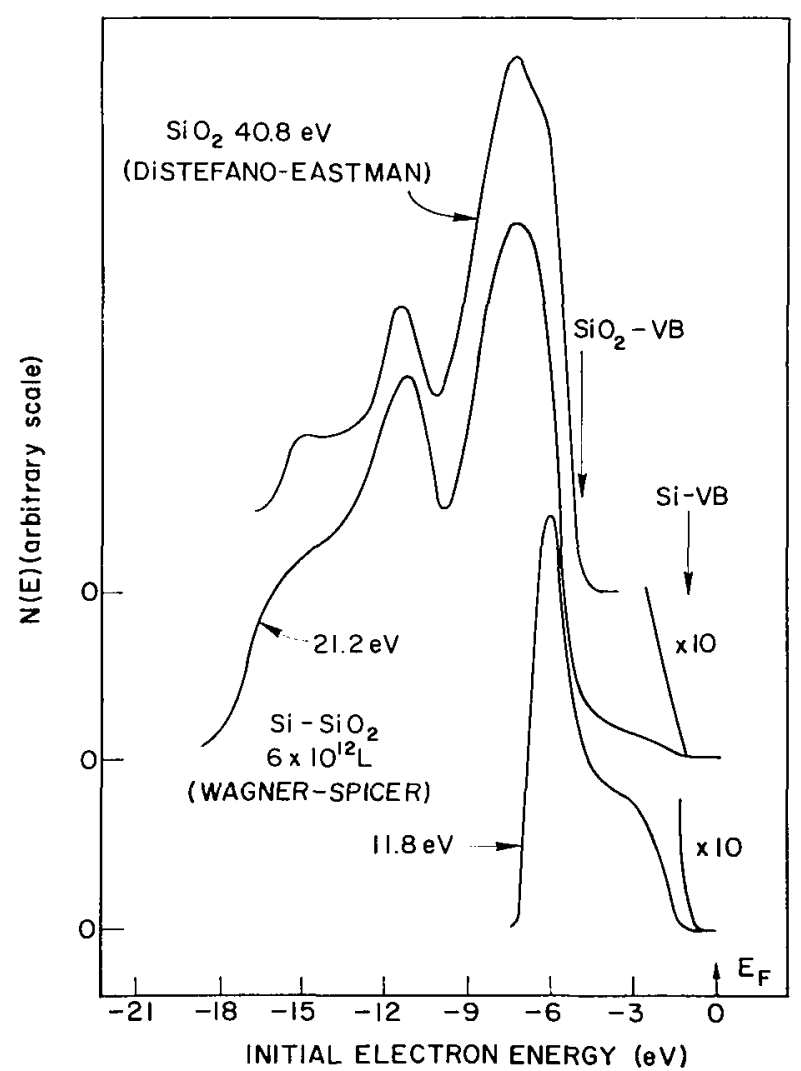

FIG. 22. - EDCs from Si with about $15 \AA$ of oxide and from quartz $\left(\mathrm{SiO}_{2}\right)$.

4. Ni results. - As is clear from the preceding, the EDCs from Ge and Si can be successfully related to the band structure of these materials using the three-step model and one-electron theory. This is not true for all materials. In figures 23 and 24, we present calculated and measured EDCs for crystalline $\mathrm{Ni}$ [44]. The calculations were done using one electron theory. Clearly there is strong disagreement between these curves. There is also difficulty in reconciling EDCs taken at various energies and relating all of this data to the calculated band structure [45], [46]. Thus, one must say that the photoemission from $\mathrm{Ni}$ cannot be presently understood using the three-step

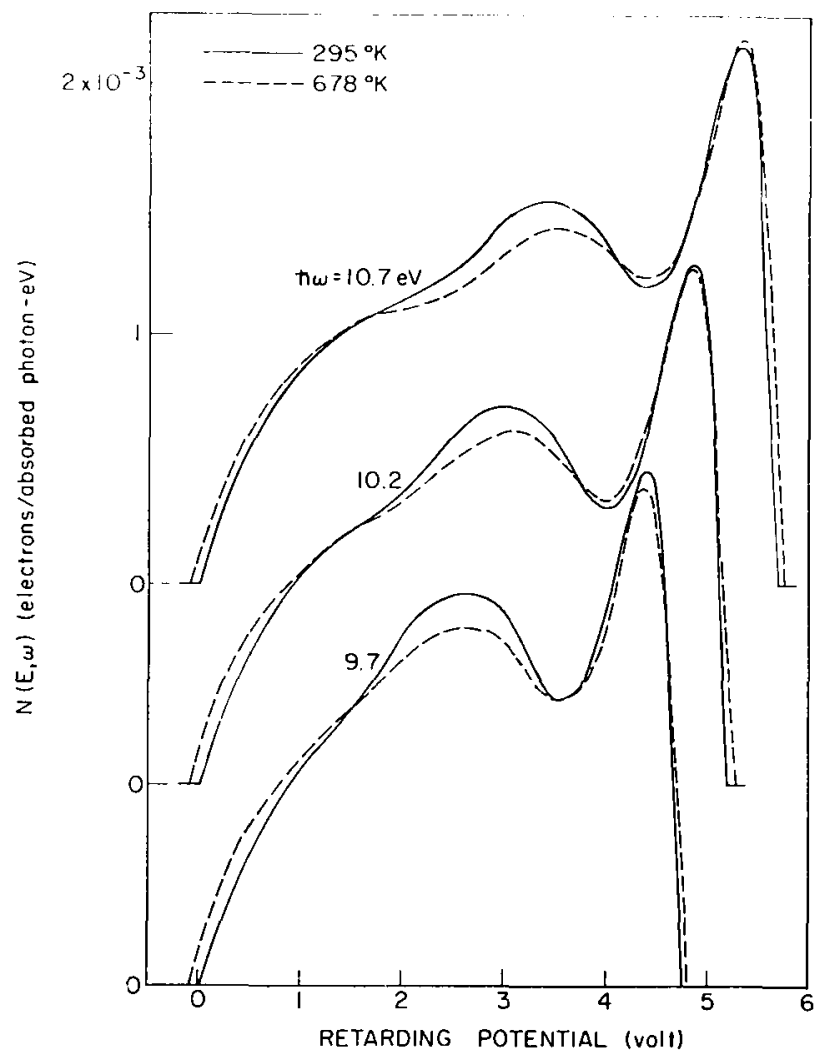

Fig. 23. - Measured EDCs from $\mathrm{Ni}$ at room temperature and above the Curie temperature.

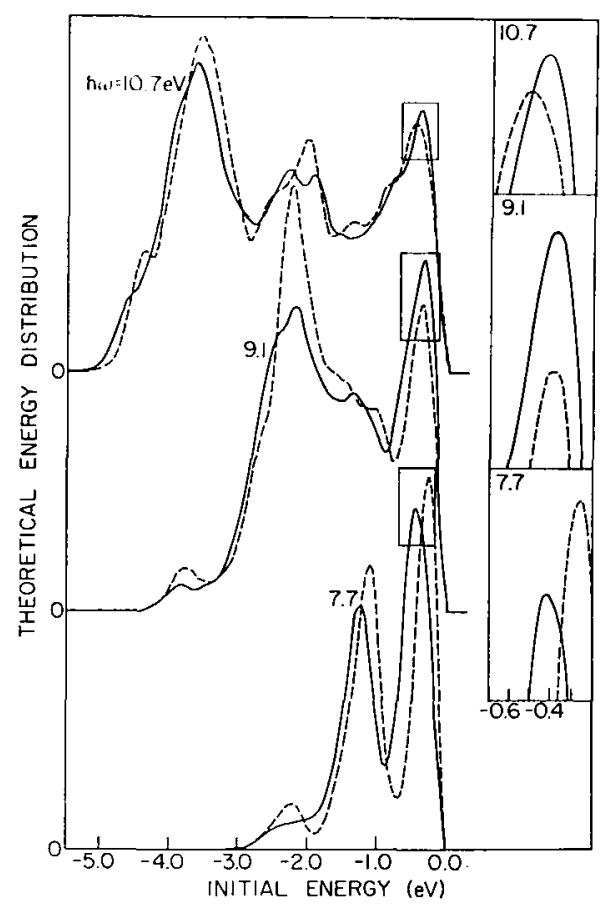

FIG. 24. - EDCs for ferromagnetic and paramagnetic Ni calculated, assuming direct transitions.

model, band structure results and assuming one electron excitation.

$\mathrm{Ni}$ is not the only example of this. The alkali [47], alkaline earth [29], [48], and divalent rare earth [49] metals show such short escape depths that the use 
of the three-step model and relating the observed EDCs to band structure in terms of one electron excitation is to be strongly questioned [29].

These examples where the three-step model can be questioned are still in a rather small minority. They are mentioned to emphasize the fact that all materials do not behave as nicely as $\mathrm{Ge}$ and $\mathrm{Si}$ and also to illustrate the need of much more work to understand the processes involved in photoemission.

5. Conclusions. - Germanium and silicon were chosen for this study because sufficient data was available to give a good overview of the use of photoemission to explore the electronic structure of these materials. For crystalline $\mathrm{Ge}$, the three-step model using band structure calculations and assuming one electron excitation with $\bar{k}$-conservation appears to work reasonably well over the complete spectral range extending from the visible through the ultraviolet into the $\mathrm{X}$-ray region. Certain questions remain without complete answers. For example, why does $k$-conservation lose its importance as an optical selection rule at photon energies near $25 \mathrm{eV}$ ? Is this due to a very short escape depth? If so, is the electron structure seen modified because of the proximity of the surface or is it identical with the bulk band structure ?
In the calculation of EDCs to compare with experiment, it is now customary to introduce only a spread or uncertainty in energy and not in $\bar{k}$. An uncertainty in $\bar{k}$ should also be introduced, particularly in cases where the escape depth becomes very short.

Using the data from $\mathrm{Si}$, it is shown how discrete surface states may be explained and defined using photoemission. It is also seen that the effect of surface contamination can be followed in photoemission and the nature of surface compounds so formed determined.

In the work on amorphous $\mathrm{Ge}$, an illustration is given of how photoemission can be used as an independent tool when no theoretical information is available on the electronic structure of a material. Again reasonable agreement is obtained between $\mathrm{X}$-ray and ultraviolet photoemission results.

Although the three-step model has been successfully used for many materials, it is pointed out that in certain classes of materials where the escape depth is very short, its use is quite questionable. It is also pointed out that there are cases where reasonable agreement cannot be obtained between photoemission results and one electron band theory. Nickel is given as an example.

\section{References}

[1] Apker, L., Taft, E. and Dickey, J., Phys. Rev. 74 (1948) 1462 ; PhilipP, H. R., TAFt, E. and APKer, L., Phys. Rev. 120 (1960) 49.

[2] Spicer, W. E. and Simon, R. E., Phys. Rev. Lett. 9 (1962) 385.

[3] Berglund, C. N. and Spicer, W. E., Phys. Rev. 136 (1964) A 1030 and A 1044.

[4] Spicer, W. E., Phys. Rev. 112 (1958) 114; J. Appl. Phys. 31 (1960) 2077.

[5] Spicer, W. E. and Berglund, C. N., Rev. Sci. Instr. 35 (1964) 1665.

[6] EdEN, R. C., Rev. of Sci. Instr. 41 (1970) 252

[7] Derbenwick, G., Pierce, D. and Spicer, W. E., " Photoemission Techniques ", in Methods of Experimental Physics ed. by Collman (Academic Press, N. Y.) to be published.

[8] Spicer, W. E. and Eden, R. C., Proc. of Ninth Intern. Conf. on Physics of Semicond. Moscow, USSR (Nauka, Leningrad, USSR, 1968) Vol. 1, p. 65.

[9] Spicer, W. E., in Electronic Density of States, ed. L. H. Bennett, Superintendent of Documents, US Government Printing Office, Washington, DC 20402 (Order by SD catalog No. C $13: 323)$ p. 139.

[10] Philipp, H. R. and Ehrenreich, H., Phys. Rev. 129 (1963) 1550.

[11] Donovan, T. M., Matsuzaki, J. and Spicer, W. E., to be published.

[12] Krolikowski, W. and Spicer, W. E., Phys. Rev. 185 (1969) 882

[13] Williams, A. R., Janak, J. F. and Moruzzi, V. L., Phys. Rev. Lett. 28 (1972) 671.

[14] Schaich, W. L. and Ashcroft, N. W., Phys. Rev. B 3 (1971) 2452.

[15] Caroli, C., Lederer-Rozenblatt, D., Roulet, B. and
Saint-James, D. (Proc. Congress du Centenaire de la Société Française de Physique), not published.

[16] Spicer, W. E. and Wooten, F., Proc. IEEE, 51 (1963) 1119 ;

Stuart, R., Wooten, F. and Spicer, W. E., Phys. Rev. 135 (1964) A 495 ;

Stuart, R. N. and Wooten, F., Phys. Rev. 156 (1967) 364.

[17] Distefano, T. H. and Spicer, W. E., Phys. Rev. B 7 (1973) 1554

[18] Brust David, Phys. Rev. 139 (1965) A 489.

[19] Mort, N. F. and Davis, E. A., Electronic Processes in Non-Crystalline Materials (Clarendon Press, Oxford) 1971.

[20] Eastman, D. E. and Grobman, W. D., Phys. Rev. Lett. 28 (1972) 1378.

[21] Wagner, L. F. and Spicer, W. E., Phys. Rev. Lett. 28 (1972) 1381

[22] WAGNER, L. F. and SPICER, W. E. (to be published).

[23] Donovan, T. M., Fischer, J. E., Matsusaki, J. and Spicer, W. E., Phys. Rev. B 3 (1971) 4292.

[24] Grobman, W. D. and Eastman, D. E., Phys. Rev. Lett. 29 (1972) 1508.

[25] Chekroun, C., Herman, F. and Ortenburger, I. (Proc. Congress du Centenaire de la Société Française de Physique). J. Physique 34 (1973) Supplt. C 6.

[26] Saravia, T. R. and Casamoyou, T., J. Phys. and Chem. Solids 32 (1971) 1075.

[27] Shay, J. L. and Spicer, W. E., Phys. Rev. 161 (1967) 799.

[28] Shay, J. L. and SpICER, W. E., Plyys. Rev. 169 (1968) 650 ; Powell, R. A., Spicer, W. E. and McMenamin, J. C., Phys. Rev. B 6 (1972) 3056.

[29] Helms, C. R. and Spicer, W. E. to be published; EASTMAN, D. E., private communication. 
[30] Aspens, D. E., Phys. Rev. Lett. 28 (1972) 913.

[31] Herman, F., Kortum, R. L., Kuglin, C. D. and Van Dyke, J. P., Methods of Computational Physics (Academic Press, New York) 1968, Vol. 8.

[32] Phillips, J. C., Phys, Rev. 125 (1962) 1931.

[33] Phillips, J. C. and Pande, K. C., Phys. Rev. Lett. 30 (1973) 787.

[34] Eastman, D. E., Freeouf, J. and Erbudak, M. (Proc. Congress du Centenaire de la Société Française de Physique). J. Physique 34 (1973) Suppl. C 6.

[35] Cohen, M. L., Bergstrasser, T. K., Phys, Rev. 141 (1966) 789

[36] Herman, F., Kortun, R. L., Kuglin, C. D. and Shap, J. L., Procs. II-VI Conf., ed. D. G. Thomas (W. A. Benjamin, N. Y.) 1967.

[37] Ley, L., Kowalczyk, S., Pollack, R. and Shirley, D. A., Phys. Rev. Lett. 29 (1972) 1088.

[38] Sicer, W. E. and Donovan, T. M., Phys, Lett. 36A (1971) 459.

[39] Donovan, T. M., Dissertation, Ph. D., Stanford University, 1970, unpublished.

Donovan, T. M. and Spicer, W. E., Phys. Rev. Lett. 21 (1962) 1572 ;

SPICER, W. E. and Donovan, T. M., J. Non-Crystalline Solids 2 (1970) 66 ;

Ribing, C. G., Pierce, D. T. and Spicer, W. E. 4 (1971) 4417:
Pierce, D. T., Ribring, C. G. and Spicer, W. E., J. NonCrystalline Solids 8-10 (1972) 959 ;

Donovan, T. M. and SPICER, W. E., to be published.

[40] Brown, F. C. and Om Rustigi, P., Phys. Rev. Lett. 28 (1972) 497.

[41] Thorpe, M. F. and Wealre, D., Phys, Rey, Lett. 27 (1971) 1581.

[42] Wagner, L. and Spicer, W. E., to be published.

[43] Distefano, T. H. and Eastman, D. E., Phys. Rev. Lett. 27 (1971) 1560.

[44] Pierce, D. T. and Spicer, W. E., Phys. Rev. B 6 (1972) 1787.

[45] Eastman, D. E., Proc. of International Conf. on Electron Spectroscopy, California, 1971 (North Holland, Amsterdam) 1972, p. 487.

[46] Hufner, S., Wertheim, G. K., Smith, N. V. and Taum, M. M., Sol. State Commun. 11 (1972) 323.

[47] SMITH, N. V., CRC Critical Reviews in Solid State Sciences 2 (1971) 45.

[48] Kress, K. A. and Lapeyre, G. J., Sol. State Commum. 9 (1971) 827.

[49] Blodgett, A. J., Jr, Spicer, W. E. and Yu, A.Y-C., in Optical Properties and Electronic Structure of Metals and Alloys, ed. F. Abeles (North Holland Pub. Co., Amsterdam) 1966, p. 246. 